\title{
Gold-Etched Silver Nanowire Endoscopy: Toward a Widely Accessible Platform for Surface-Enhanced Raman Scattering-Based Analysis in Living Cells
}

\author{
Monica Ricci, Beatrice Fortuni,* Raffaele Vitale, Qiang Zhang, Yasuhiko Fujita, Shuichi Toyouchi, \\ Gang Lu, Susana Rocha, Tomoko Inose, and Hiroshi Uji-i*
}

Cite This: https://dx.doi.org/10.1021/acs.analchem.0c04120

Read Online

ACCESS | Lلll Metrics \& More | 国 Article Recommendations | (1) Supporting Information

\begin{abstract}
Recently, our group introduced the use of silver nanowires (AgNWs) as novel non-invasive endoscopic probes for detecting intracellular Raman signals. This method, although innovative and promising, relies exclusively on the plasmonic waveguiding effect for signal enhancement. It, therefore, requires sophisticated operational tools and protocols, drastically limiting its applicability. Herein, an advanced strategy is offered to significantly enhance the performance of these endoscopic probes, making this approach widely accessible and versatile for cellular studies. By uniformly forming gold structures on the smooth AgNW surface via a galvanic replacement reaction, the density of the light coupling points along the whole probe surface is drastically increased, enabling high surface-enhanced Raman

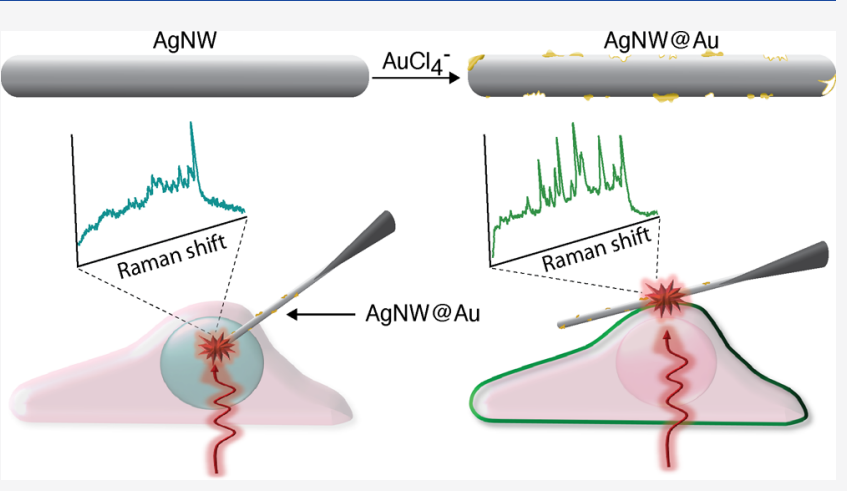
scattering (SERS) efficiency upon solely focusing the excitation light on the gold-etched AgNW. The applicability of these goldetched AgNW probes for molecular sensing in cells is demonstrated by detecting site-specific and high-resolved SERS spectra of cell compartment-labeling dyes, namely, 4',6-diamidino-2-phenylindole in the nucleus and 3,3'-dioctadecyloxacarbocyanine on the membrane. The remarkable spectral sensitivity achieved provides essential structural information of the analytes, indicating the overall potential of the proposed approach for cellular studies of drug interactions with biomolecular items.
\end{abstract}

$\mathrm{T}$ he ability of unraveling drug dynamics down to the single-cell level with high spatiotemporal resolution is among the ultimate goals in nanomedicine. ${ }^{1-5}$ Tremendous efforts have been put in increasing therapeutic drug specificity, boosting their efficacy and defeating drug resistance while decreasing their devastating side effects. ${ }^{6,7}$ Key to success of these ambitions is a better understanding of both drug intracellular distribution and action mechanism. ${ }^{8-10}$ To this end, surface-enhanced Raman scattering (SERS) holds excellent potential. The high sensitivity and fingerprinting capabilities of this method allow to also observe nonfluorescent molecules, identify their structural conformation and molecular interactions, and even monitor dynamical changes. ${ }^{11,12}$ SERS measurements in living cells are usually performed via endocytosed metal nanoparticles (NPs). Despite this being the most commonly used approach, it suffers from significant limitations such as poor spatiotemporal control and irreversible cellular uptake of the NPs. As a consequence of these shortcomings, interest has risen toward the development of 1D "nano-endoscopes", which can be physically inserted into live cells to target local spots. ${ }^{13-15}$ The pioneer works performed in this context rely on conical-shaped silver nanopipettes or glass pipettes coated with noble metal
NPs. ${ }^{14-18}$ Although diameters as small as $100 \mathrm{~nm}$ have been reported at the tip, the large apex angle and rigid structure confer significant damage to the cell health once the tip is inserted, altering the outcomes of the investigations performed. ${ }^{19}$ Recently, carbon nanotubes decorated with gold NPs have been elegantly designed. ${ }^{13,20}$ Although outstanding, this approach requires elaborate fabrication protocols for obtaining an ideal NP decoration for high SERS enhancements while retaining a suitable probe diameter for a non-invasive insertion in cells. ${ }^{19}$ Taken together, these stringent requirements call for alternatives, which can be found in SERS-active noble metal nanowires (NWs).

In this regard, we previously designed a silver NW (AgNW)based endoscopic probe as a non-invasive cutting-edge tool for the detection of intracellular SERS signals. ${ }^{21}$ After inserting the

Received: September 30, 2020

Accepted: January 19, 2021 
a)
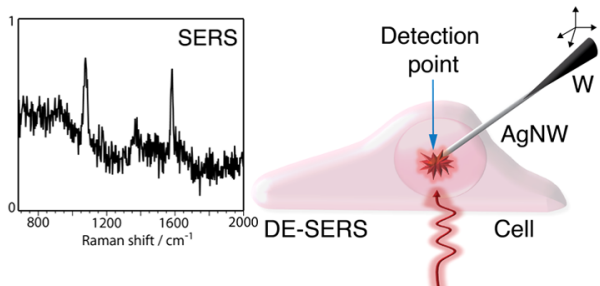

c)

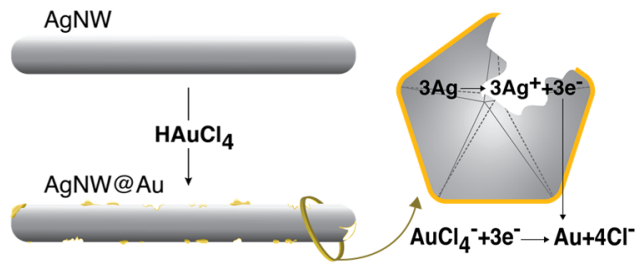

e)
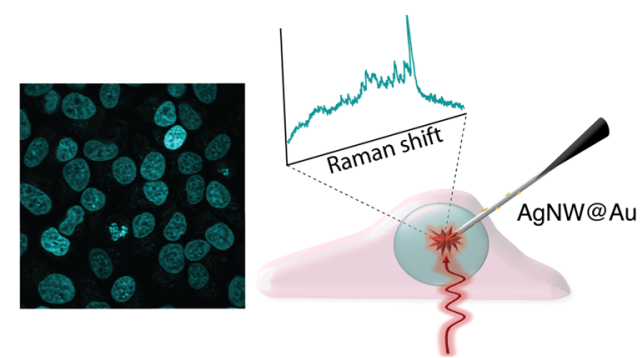

b)

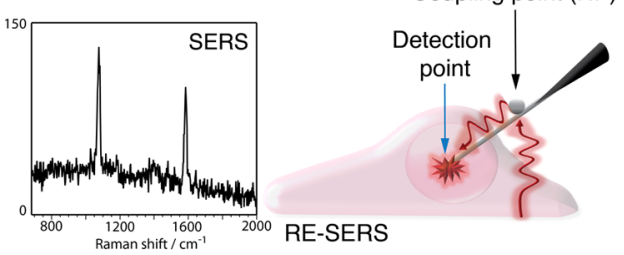

d)

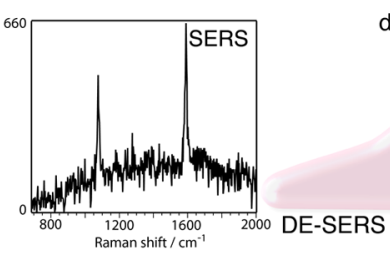

Multiple detection points

f)

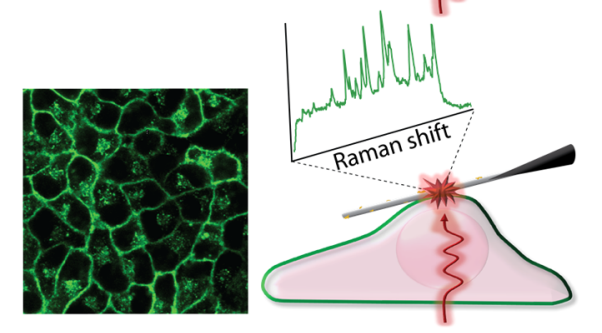

Figure 1. Original endoscopy technique, improvement and proof of concept. (a,b) Schematic representation of the AgNW-based SERS endoscopy technique applied with (a) direct laser excitation (DE-SERS) and (b) remote configuration (RE-SERS) (original technique). ${ }^{21}$ (c) Chemical and morphological changes induced by the galvanic replacement reaction (GRR) and yielding gold-etched AgNWs (AgNW@Au). (d) AgNW@Aubased SERS endoscopy technique. SERS spectra in $(\mathrm{a}, \mathrm{b}, \mathrm{d})$ are given for illustrative purposes and correspond to the 4-mercaptobenzoic acid (4MBA) SERS spectra obtained by in vitro characterizations. (e,f) Schematic representation of the AgNW@Au-based endoscopy of the nucleus (e) and the cell membrane (f) of a single living cell.

AgNW probe into a single living cell, the laser was focused at the AgNW tip, and SERS signals were recorded from the same position (direct excitation, DE-SERS) (Figure 1a). While the direct excitation configuration generated a limited SERS enhancement, the application of remote excitation (RESERS, Figure 1b) enabled the acquisition of SERS spectra with lower background and higher signal-to-noise ratio. Despite its innovative character, this latter geometry, where the laser light is focused at the middle of AgNWs, several micrometers away from the detection point (the tip of the NW), is hardly reproducible with conventional optical microscopes. Moreover, the fabrication of remote SERS-active probes is time consuming and nontrivial, even for expert operators (see also the Supporting Information).

Here, we bring our AgNW technique to the next level, making it widely applicable, easily accessible, and extremely sensitive for detecting trace-level analytes. To this purpose, the SERS activity of the $1 \mathrm{D}$ endoscopic probes was dramatically improved by simply subjecting the AgNWs to a GRR. The GRR represents a simple way to modify the NW surface through electrochemical silver oxidation and gold reduction, which ultimately leads to gold deposition and silver etching, valuable hotspots for higher SERS enhancements (Figure 1c). ${ }^{22,23}$ The uniform hotspot distribution obtained after GRR guarantees the collection of well-resolved signals over the whole length of the endoscopic probe (Figure 1d). All these features are crucial to carry out high-throughput cellular investigations, as they permit to collect SERS signals upon simple direct excitation and perform multiple SERS detections from different regions upon a single probe placement. Ultimately, we demonstrate the site specificity and applicability of these probes for the detection of low-concentration (tracelevel) exogenous molecules by obtaining highly resolved fingerprints of target molecules (organic dyes) located in the nucleus (Figure 1e) and on the plasma membrane of living cells (Figure 1f). The remarkable spectral sensitivity achieved further enabled to extract essential structural information of the compounds under investigation.

\section{MATERIALS AND METHODS}

Materials. Silver nitrate $\left(\mathrm{AgNO}_{3}, 99.9999 \%\right)$, polyvinylpyrrolidone (PVP) $\left(M_{\mathrm{w}} \sim 40,000\right)$, copper chloride $\left(\mathrm{CuCl}_{2}\right.$, 99.9\%), ethylene glycol (EG, anhydrous, 99.8\%), chloroauric acid $\left(\mathrm{HAuCl}_{4}, 99.9999 \%\right)$, and 4-MBA (99\%) were purchased from Sigma-Aldrich. 2-Propanol (IPA, $\geq 99.5 \%$ ) and sodium hydroxide $(\mathrm{NaOH}, 10 \mathrm{M})$ were purchased from VWR Chemicals. Tungsten $(\mathrm{W})$ and platinum $(\mathrm{Pt})$ wire were provided by Advent Research Materials. Epoxy glue was purchased from RS components. Dulbecco's modified Eagle's medium (DMEM), gentamicin, Dulbecco's phosphate-buffered saline (no calcium, no magnesium), trypsin-EDTA $(0.5 \%, 10 \times)$, Hank's balanced salt solution (HBSS, no phenol red), GlutaMax supplement, fetal bovine serum (FBS, South America origin), 4',6-diamidino-2-phenylindole (DAPI) celllabeling solution, and Vybrant 3,3'-dioctadecyloxacarbocyanine (DiO) cell-labeling solution were purchased from Thermo Fisher Scientific. All the chemicals were used without further purifications. 
AgNW Synthesis. AgNWs were synthesized following the polyol method, as previously reported. ${ }^{21,24}$ Briefly, $6.5 \mathrm{~mL}$ of EG containing PVP $(0.1 \mathrm{M})$ were first heated at $160{ }^{\circ} \mathrm{C}$ for 1 $\mathrm{h}$, followed by addition of $80 \mu \mathrm{L}$ of $\mathrm{CuCl}_{2}(4 \mathrm{mM})$. After 10 min, $1.5 \mathrm{~mL}$ of $\mathrm{AgNO}_{3}$ solution $(130 \mathrm{mM})$ in EG was added dropwise at a typical rate of $300 \mu \mathrm{L} / \mathrm{min}$. The final solution was kept under magnetic stirring at $160{ }^{\circ} \mathrm{C}$ for an additional 2 $\mathrm{h}$ in order to obtain an average NW length of $15 \mu \mathrm{m}$. After the synthesis, the AgNWs were kept in EG to prevent oxidation.

Galvanic Replacement Reaction. Room temperature. GRR was performed with different $\mathrm{HAuCl}_{4}$ concentrations in order to find the best conditions for obtaining the highest SERS enhancement. The concentrations of $\mathrm{Au}^{3+}$ investigated ranged from 10 to $75 \mu \mathrm{M}$. Higher concentrations of $\mathrm{Au}^{3+}$ were also investigated, but led to AgNWs precipitation, and were therefore excluded. The detailed protocol follows. PVP was first dissolved in Milli-Q water at a concentration of $1 \mathrm{mg} / \mathrm{mL}$, which was kept constant for each sample. The presence of PVP is supposed to prevent the precipitation of AgNWs, especially at high $\mathrm{Au}^{3+}$ concentrations. Once the PVP was completely dissolved, aqueous solutions of $\mathrm{HAuCl}_{4}$ were added to each sample, obtaining final $\mathrm{Au}^{3+}$ concentrations of 10,25, 50, and $75 \mu \mathrm{M}$. Afterward, $100 \mu \mathrm{L}$ of AgNWs, from synthesis, was added dropwise under vigorous stirring to each of the solutions containing chloroauric acid. The samples were kept under vigorous stirring at room temperature for $3 \mathrm{~h}$. Afterward, the AgNWs@Au were washed three times with Milli-Q, in order to remove the PVP molecules, and other three times with IPA. The resulting AgNWs@Au were characterized by transmission electron microscopy (TEM) (Figure S1). $80{ }^{\circ} \mathrm{C} .15 \mathrm{~mL}$ of Milli-Q water was refluxed at $80{ }^{\circ} \mathrm{C}$ for $10 \mathrm{~min}$. Afterward, 450 $\mu \mathrm{L}$ of $\mathrm{AgNW}$ solution in EG (directly from synthesis) was added to the solution, which was then mixed to $1.8 \mathrm{~mL}$ of $\mathrm{HAuCl}_{4}$, obtaining final $\mathrm{Au}^{3+}$ concentrations of 10, 20, 40, and $60 \mu \mathrm{M}$. The mixture was then refluxed for other $15 \mathrm{~min}$. Vigorous magnetic stirring was maintained during the entire process. Subsequently, the reaction solution was washed three times with IPA and re-dispersed in IPA. The resulting AgNWs@Au were characterized by TEM (Figure S2). To investigate the SERS enhancements, SERS mapping was carried out after functionalization with 4-MBA (Figure S3). Spectra averaged over the individual maps were extracted for each concentration for comparison (Figure S4).

SERS Characterization. In order to investigate the AgNWs@Au SERS performances, the NW surface was first functionalized with 4-MBA, a standard Raman reporter. The IPA solution containing $50 \mu \mathrm{L}$ of AgNWs@Au and 4-MBA (final concentration $2 \mathrm{mM}$ ) was kept under stirring overnight. Afterward, the AgNWs@Au were washed three times with IPA via centrifugation and spin-coated onto a clean glass coverslip. SERS maps were obtained by scanning point-by-point the AgNW@Au surface with a 633 nm laser (integration time: $1 \mathrm{~s}$, circular polarization). Details related to the SERS apparatus are reported in the section concerning the endoscopy experiments.

Enhancement Factor Calculation. The enhancement factor (EF) was estimated based on the following equation

$$
\mathrm{EF}=\frac{\left(I_{\mathrm{SERS}} / N_{\mathrm{SERS}}\right)}{\left(I_{\text {bulk }} / N_{\text {bulk }}\right)}
$$

where $I_{\text {SERS }}$ and $I_{\text {bulk }}$ are the SERS intensity of 4-MBA from the surface of the AgNWs@Au and that of normal Raman scattering from 4-MBA, respectively. $N_{\text {SERS }}$ and $N_{\text {bulk }}$ are the number of 4-MBA molecules. Full coverage of 4-MBA monolayers on the AgNWs@Au with $0.8 \times 0.8 \mathrm{~nm}^{2}$ spacing per molecule was assumed and the diameters of AgNWs@Au were estimated to be $150 \mathrm{~nm}$. The number of 4-MBA molecules $N_{\text {bulk }}$ was $6.07 \times 10^{8}$, and the number of 4-MBA molecules $N_{\text {SERS }}$ was $4.9 \times 10^{5}$. Considering the laser power $(61.8 \mu \mathrm{W})$, the maximum EF calculated for AgNWs@Au was $3.5 \times 10^{5}$.

Endoscopy Probe Preparation. A W tip of diameter 50 $\mu \mathrm{m}$ was etched by applying a DC voltage, with frequency 50 $\mathrm{Hz}$ and amplitude $3 \mathrm{~V}$, between the $\mathrm{W}$ wire and a ring-shaped Pt wire electrode of diameter $8 \mathrm{~mm}$ in a $2 \mathrm{M}$ aqueous solution of $\mathrm{NaOH}$. After rinsing with Milli-Q water, the so-obtained sharp W tip was immersed in IPA containing AgNWs@Au diluted 1:100, v/v. The attachment of the AgNWs on the W wire was performed by alternating current (AC) dielectrophoresis method. An AC voltage with a frequency of $1 \mathrm{MHz}$ and an amplitude of $8 \mathrm{~V}$ was applied between the $\mathrm{W}$ tip and the ring-shaped $\mathrm{Pt}$ wire electrode, and after $c a .5 \mathrm{~s}$, the tip was retracted from the solution (keeping the voltage applied). The probe was then annealed at $250^{\circ} \mathrm{C}$ for $15 \mathrm{~min}$ on a hotplate for steadying the bond. Subsequently, the junction between the AgNWs@Au and the W was glued with a conductive epoxy using a motorized four-axis micromanipulator (MX7600, Siskiyou) equipped with an epoxy pre-coated etched $\mathrm{W}$ tip on an optical microscope (Ti-U, Nikon), and a $40 \times$ objective (N.A. 0.6, Nikon). The AgNW@Au probe (Figure S6) was then used in live-cell endoscopy experiments.

Cell Culture. HeLa cells were cultured in $25 \mathrm{~cm}^{2}$ cell culture flasks at $37{ }^{\circ} \mathrm{C}$ and in a humidified $5 \% \mathrm{CO}_{2}$ atmosphere. The cell passage was performed via trypsinization when the confluency reached $80 \%$. The cell culture was maintained in DMEM containing 10\% FBS, 1\% L-GlutaMax, and $0.1 \%$ gentamicin. For imaging, cells were cultured in 35 $\mathrm{mm}$ glass bottom dishes (MatTeK).

Live-Cell SERS Endoscopy Experiments. Before probe insertion, the cell sample was washed three times with $1 \mathrm{~mL}$ of HBSS and the glass bottom dish was then placed on an inverted microscope ( $\mathrm{Ti}-\mathrm{U}$, Nikon) equipped with a piezoelectric stage (P517.3CL, Physik Instrument). The AgNW@ $\mathrm{Au}$ probe was slowly inserted into the nucleus of a live $\mathrm{HeLa}$ cell with diagonal movements, keeping an angle of about $30^{\circ}$. Measurements in the plasma membrane were carried out by bringing the AgNW@Au probe above the living cell and by gently moving the probe down until the endoscopy probe reached the cellular membrane (angle about $45^{\circ}$ ). Both movements were controlled by a motorized four-axis micromanipulator (MX7600, Siskiyou). For SERS measurements, continuous wave $632.8 \mathrm{~nm} \mathrm{He}-\mathrm{Ne}$ laser (Research ElectroOptics) was reflected by a dichroic mirror (Z633RDC, Chroma) and was then focused on the AgNWs@Au by an objective lens (Plan Fluor 60×, N.A. 0.85, Nikon). Circular polarization at the sample was achieved by tuning halfwavelength $(\lambda / 2)$ and quarter-wavelength $(\lambda / 4)$ wave plates. Raman scattering light was collected from the same focal point and guided to a spectrograph (iHR320, HORIBA) equipped with a cooled charge-coupled device (CCD) (Newton 920P, Andor) after passing through a confocal pinhole (diameter 100 $\mu \mathrm{m}$ ) and a longpass filter (HQ645LP, Chroma). Typically, the laser power used was $1 \mathrm{~mW}$, and the integration time applied was $1 \mathrm{~s}$ [waterfall (WF) measurements].

$\mathrm{Ca}^{2+}$ Response Studies. Samples for cell stress tests were prepared by culturing HeLa cells in $35 \mathrm{~mm}$ glass bottom dishes 

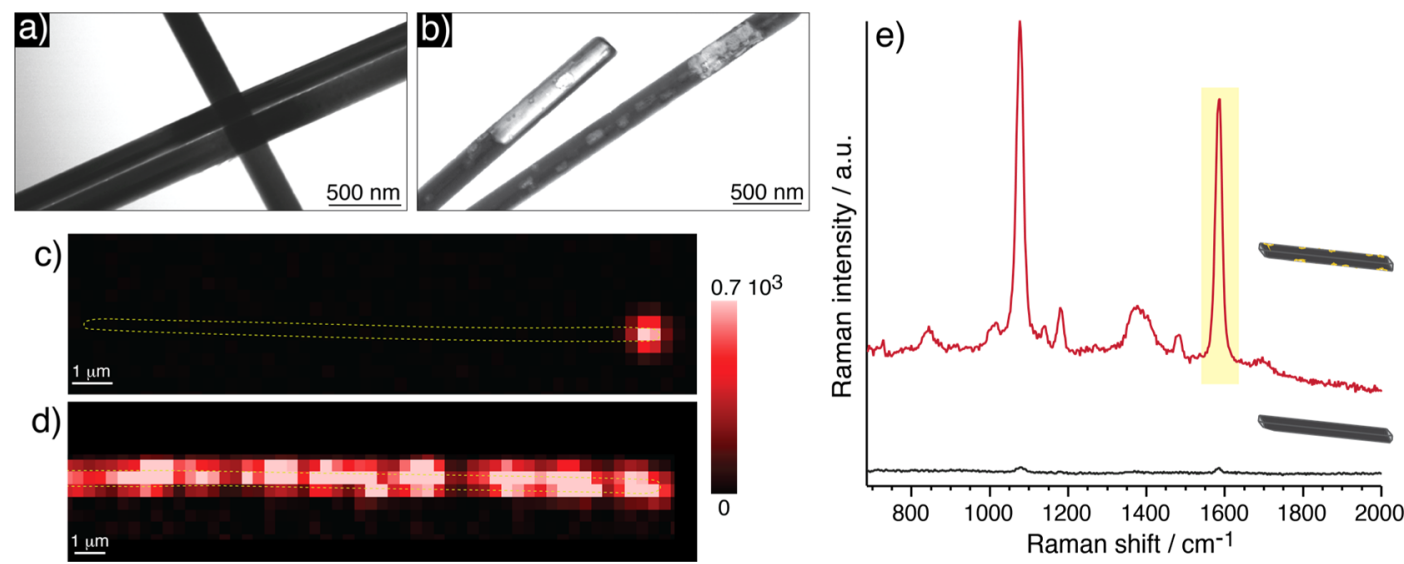

Figure 2. Characterization and SERS performances of AgNWs@Au. (a,b) TEM images of AgNWs before (a) and after (b) GRR (AgNWs@Au). (c,d) 4-MBA SERS mapping images of AgNW (c) and AgNW@Au (d) generated for the $v(\mathrm{CC})$ ring breathing mode at $1587 \mathrm{~cm}^{-1}$ (yellow background in (e)). The NW area is highlighted by yellow dashed lines. (e) Average 4-MBA SERS spectra of an AgNW@Au (top) and an AgNW (bottom), respectively. $\lambda_{\text {exc }}: 633 \mathrm{~nm}$; power: $61.8 \mu \mathrm{W}$; and polarization: circular.

(MatTeK). When the confluency reached about $60 \%$, the cell sample was washed three times with $1 \mathrm{~mL}$ of HBSS and stained with Calcium Green-1 AM (Invitrogen). Briefly, a solution containing the calcium indicator $(5 \mu \mathrm{M})$ and Cremophor EL $(0.01 \% \mathrm{w} / \mathrm{v})$ was added to the cell sample in HBSS. The cells were incubated at room temperature for $30 \mathrm{~min}$ and washed three times with HBSS before imaging. Fluorescence images during probe insertion and release were obtained using the set up described above for live-cell endoscopy measurements. Wide-field illumination was provided by a $488 \mathrm{~nm}$ laser (Stabilite 2018, Spectra-Physics).

Cellular Staining. Samples for cellular staining were prepared by culturing HeLa cells in $35 \mathrm{~mm}$ glass bottom dishes (MatTeK). When the confluency reached about $60 \%$, the cell sample was washed three times with $1 \mathrm{~mL}$ of HBSS and stained as follows. The nucleus of HeLa cells was stained with DAPI, while the cellular membrane was stained with $\mathrm{DiO}$, individually.

Briefly, $300 \mu \mathrm{L}$ of DAPI $(2.5 \mu \mathrm{M})$ or $\mathrm{DiO}(8.3 \mu \mathrm{M})$ in HBSS was added to the cell sample after removal of the cell medium. After $5 \mathrm{~min}$ of incubation with the dye, the cell dish was washed three times with $1 \mathrm{~mL}$ of HBSS. The cells were then kept in HBSS for being imaged by a confocal fluorescence microscope (FV1000, Olympus) or investigated via endoscopy.

SERS Measurements In Vitro. To obtain the SERS spectra in vitro, the AgNWs@Au were spin-coated onto a clean glass coverslip, heated up $80{ }^{\circ} \mathrm{C}$ to fix the NWs onto the glass surface, and functionalized with the molecule of interest. SERS reference measurements were carried out for free DAPI, free $\mathrm{DiO}$, and DNA-DAPI complex. To obtain the SERS spectrum of DAPI (or DiO), the solution containing DAPI $(2.5 \mu \mathrm{M})$, or $\mathrm{DiO}(8.3 \mu \mathrm{M})$, was drop-cast and left onto the glass coverslip overnight to let the molecule interact with the metal surface of the AgNWs@Auvia metal-ligand interactions. After 24 h, the coverslip was rinsed with Milli-Q water and dried with argon flux. For the SERS measurements of DNA-DAPI complex, the DNA sample was obtained extracting the nuclear DNA from HeLa cells by using the QIAamp DNA Mini Kit. During the procedure, RNAse was added to purify the DNA from RNA traces. The extracted DNA was then dispersed in Milli-Q water obtaining a solution of $244 \mu \mathrm{g} / \mathrm{mL}$. The AgNWs@Au were first functionalized with cysteamine, which will act as a linker to anchor the DNA to the NWs. The thiol groups of cysteamine molecules are known to spontaneously form covalent bonds with the metal surface of the $\mathrm{NWs}^{25}$ while the protonated amino group on the opposite site of the molecule can electrostatically interact with the DNA (negatively charged). An aqueous solution of cysteamine (1 $\mathrm{mM}$ ) was drop-cast on the spin-coated AgNWs@Au and left it to interact for $3 \mathrm{~h}$. After rinsing with Milli-Q water, the extracted DNA solution was drop-cast and incubated for $24 \mathrm{~h}$. Afterward, the coverslip was rinsed with Milli- $Q$ water, dried with argon flux, and the DAPI solution was drop-cast onto the coverslip. The dye was then allowed to interact with the DNA overnight and consequently form DAPI-DNA complexes. After $24 \mathrm{~h}$, the coverslip was again rinsed gently but consistently with Milli- $Q$ water in order to remove traces of free dye.

Pearson Correlation Coefficient. The Pearson correlation coefficient (PCC) between two spectra was estimated according to the following equation

$$
r_{x, y}=\frac{\sum_{n=1}^{N}\left(x_{n}-\bar{x}\right)\left(y_{n}-\bar{y}\right)}{\sqrt{\sum_{n=1}^{N}\left(x_{n}-\bar{x}\right)^{2}} \sqrt{\sum_{n=1}^{N}\left(y_{n}-\bar{y}\right)^{2}}}
$$

where $N$ denotes the total number of considered Raman shifts, $x_{n}$ and $y_{n}$ represent the Raman intensities registered for the first and second spectrum, respectively, at the $n$-th Raman shift, and $\bar{x}$ and $\bar{y}$ are the mean intensity values recorded for the first and second spectrum, respectively. $r_{x, y}$ can be regarded as a measure of the overall similarity between the two profiles under study. In order to obtain an unbiased estimation of the PCC, each pair of spectra was preliminarily baseline-corrected by asymmetric least squares. ${ }^{26}$

\section{RESULTS AND DISCUSSION}

Method. AgNWs were synthesized as reported previously ${ }^{21}$ and subjected to GRR for increasing the hotspots' density along the surface of the nanoprobe. GRR was performed by simply mixing an aqueous solution of tetrachloroauric acid $\left(\mathrm{HAuCl}_{4}\right)$ with a colloidal AgNW solution under vigorous stirring, yielding AgNWs decorated with gold structures (AgNWs@Au, more details in the Supporting Information). Different temperatures (RT and $80{ }^{\circ} \mathrm{C}$ ) and $\mathrm{Au}^{3+}$ concen- 
a)

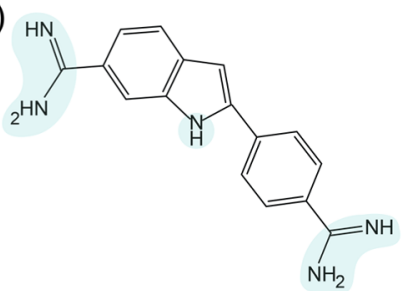

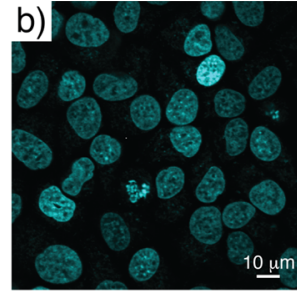

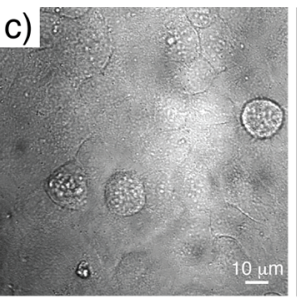

d)

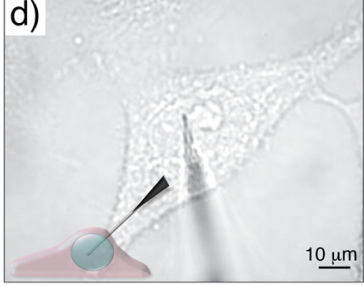


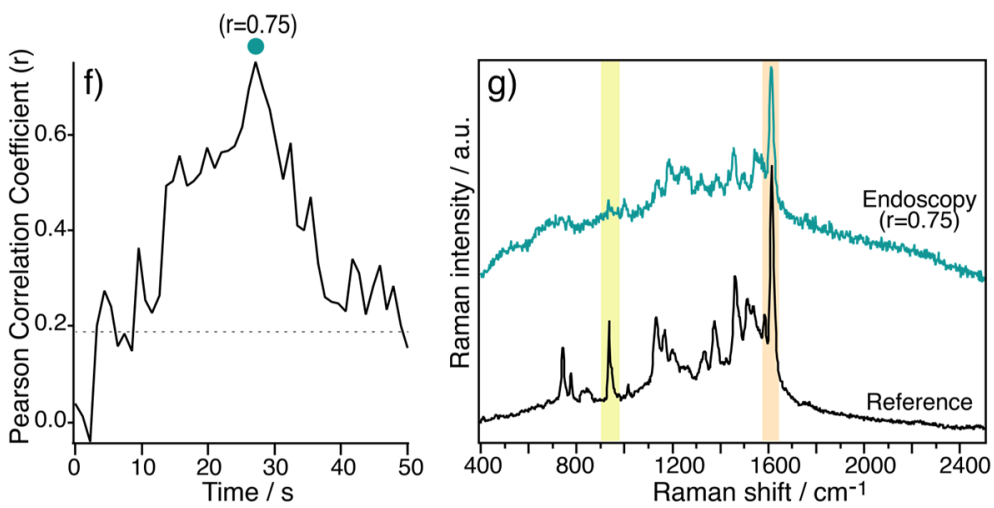

Figure 3. SERS endoscopy from the nucleus of a DAPI-stained HeLa cell. (a) Chemical structure of DAPI, with groups interacting with DNA highlighted in blue. $(b, c)$ Confocal fluorescence (b) and transmission (c) image of HeLa cells stained with DAPI. (d) Transmission image of the endoscopy probe upon insertion into the cell nucleus of a single HeLa cell. Inset: endoscopy experiment schematics. (e) Representative WF plot featuring SERS spectra of DAPI from the cell nucleus over time. (f) Time trend of the PCCs calculated between each spectrum of the WF shown in (e) and the DAPI SERS reference spectrum (in vitro). The horizontal line denotes the 95th percentile of the PCC distribution obtained for the control experiment $(r=0.19)$. Statistically significant $r$ values can be found above the percentile line. $(\mathrm{g})$ SERS spectra of DAPI from endoscopy measurements (top) and from in vitro measurements (reference) (bottom). Relevant Raman band changes are highlighted in yellow and orange.

trations $(10-75 \mu \mathrm{M})$ were tested for getting the best GRR products to be used for SERS-based endoscopy in living cells (Figures $\mathrm{S} 1-\mathrm{S} 4$ and relative discussion). Among these tests, AgNWs@Au obtained from GRR at $80{ }^{\circ} \mathrm{C}$ with $20 \mu \mathrm{M}$ of $\mathrm{Au}^{3+}$ provided the highest SERS performance (high signal enhancement and high density of hotspots), while maintaining a suitable morphology for live cell endoscopy (Figure 2). TEM images of AgNWs before and after GRR (AgNWs@Au) displayed evident alterations in the topographic features without drastic changes in the NW morphology. While a smooth surface was observed for typical as-synthesized AgNWs (Figure 2a), gold-deposited nanostructures and etched regions were evident in the AgNWs@Au (bright parts in Figure 2b).

To evaluate the improvement of the SERS performances after GRR, AgNWs and AgNWs@Au were functionalized with a commonly used Raman reporter, 4-mercaptobenzoic acid (4MBA), and SERS intensity maps over the entire NW area were collected. Figure $2 c, d$ shows the intensity distribution of the 4MBA Raman peak at $1587 \mathrm{~cm}^{-1}$ (yellow background in Figure 2e) for an AgNW before and after GRR $\left(80{ }^{\circ} \mathrm{C}, 20 \mathrm{mM}\right)$, respectively. The SERS map on the untreated AgNW revealed signal enhancement only at the NW apex. In strong contrast, the SERS map on the AgNW@Au showed a high density of hotspots, intense and uniformly distributed along the entire surface. Spectra recorded from three different locations on the AgNW@Au map are reported in Figure S5.

To demonstrate the high reproducibility of the hotspot formation, SERS mapping images of AgNWs@Au coming from different reaction batches are displayed in Figure S6. The higher SERS activity of AgNWs@Au was further confirmed by comparing the 4-MBA SERS spectra averaged over the entire SERS maps of the NW before and after the GRR reaction (Figure 2e). The averaged 4-MBA spectrum extracted from the
AgNW map (Figure 2c) led to a very low signal-to-noise ratio (black line in Figure 2e). Conversely, the averaged SERS fingerprint of 4-MBA obtained from the AgNW@Au map (Figure 2d) reveals a high signal-to-noise ratio and wellresolved Raman spectrum (red line in Figure 2e), clearly showing the two specific peaks at 1078 and $1587 \mathrm{~cm}^{-1}$, attributed to the benzene ring breathing mode. ${ }^{27}$ The typical averaged EF of SERS on AgNW@Au was estimated to be in the order of $10^{5}$ (calculation details reported in the Supporting Information).

Besides the uniform hotspot distribution observed for AgNWs@Au, which allows signal collection over the whole length of the endoscopic probe in high throughput and accessible fashion, the remarkable SERS sensitivity achieved enables recording resolved spectra at very short acquisition time (e.g., $1 \mathrm{~s}$ ) and, therefore, obtaining an extensive data set in a concise experimental timeframe.

The overall potential of the AgNWs@Au toward analysis in cells was then tested via SERS-based endoscopy. The endoscopic probes were prepared by attaching the AgNWs@ $\mathrm{Au}$ onto a pre-etched tungsten tip (Figure S7), in turn, anchored to a 4-axis micromanipulator on the microscope. ${ }^{21}$ In order to assess whether AgNWs@Au retain low invasiveness, the cellular stress induced by AgNW@Au insertion was investigated by monitoring changes in the concentration of cytoplasmic calcium ions $\left(\mathrm{Ca}^{2+}\right)$. The live cells were stained with Calcium Green, an indicator which emits strong fluorescence upon binding with $\mathrm{Ca}^{2+}$. While no variation in the fluorescence signal was observed upon insertion and release, of an AgNW@Au endoscopic probe (Figure S8a-d), a strong increase in the fluorescence signal was observed when a tungsten tip (W) (positive control) was used (Figure S8e-g). 
To demonstrate the suitability of AgNW@Au for the intracellular investigation of exogenous molecules, such as therapeutic drugs, we applied these probes for the identification of molecules inside living HeLa cells. As target molecules, fluorescent dyes were used. Cell-labeling dyes represent a good study model thanks to their (i) easy monitoring through fluorescence, (ii) high Raman cross sections, (iii) specificity toward given cellular compartments, and (iv) sizes comparable to that of therapeutic drugs. The nuclear labeling dye DAPI was chosen as a model study for therapeutic drugs, which normally target the cellular machinery inside the nucleus. ${ }^{28,29}$ Concurrently, the membrane labeling $\mathrm{DiO}$ was selected as a representative for high-interest therapeutic agents targeting the plasma membrane. ${ }^{30-32}$ Accordingly, prior to the endoscopy experiments, the nucleus or plasma membrane were labeled with DAPI or DiO, respectively. Investigations through Raman spectroscopy require reference spectra of the compounds under study, to be compared with the spectra obtained via endoscopy. In the light of this, fingerprints of the same dyes were recorded by adsorbing them on AgNWs@Au, previously spin-coated onto a glass coverslip (more details in the Supporting Information). The latter measurements will be defined as in vitro over the remaining manuscript to be distinguished from the ones collected via endoscopy, which were performed in living cells (in cellulo).

Nucleus. The cellular nucleus of HeLa cells was stained with DAPI, a common fluorescent marker which binds externally to the DNA through hydrogen bonds with the indole- $\mathrm{NH}$ proton and the amidine groups (Figure 3a, blue background). ${ }^{33-35}$ Fluorescence and transmission images of DAPI-stained HeLa cells are displayed in Figure 3b,c, respectively. This sample was then used for endoscopy measurements. Following the approach shown in Figure $1 b$, an AgNW@Au probe was carefully inserted into the nucleus of a stained living HeLa cell (Figure 3d, Movie S1, and Figure S9) and, by focusing a $633 \mathrm{~nm}$ laser beam on the probe apex (located inside the nucleus), a series of SERS spectra were collected over time ( $1 \mathrm{~s}$ integration time). Figure $3 \mathrm{e}$ shows a representative WF plot of the SERS spectrum of DAPI over a time period of $50 \mathrm{~s}$. It is important to note that each WF collected during the endoscopy measurements contains a series of spectra coming from any molecule in the proximity of the NW. Therefore, considering the heterogeneity and dynamics of the cellular environment, and the absence of any targeting functionalization on our probes, the data set did not exclusively contain the SERS spectra of the target molecules (in this case, DAPI).

To identify the SERS spectra of DAPI among the multitude of spectra collected, we applied PCC analysis, commonly used for SERS spectral analysis of big data sets. ${ }^{36,37}$ The PCC value (denoted by $r$ ) provides an estimation of the similarity between every individual endoscopy spectrum acquired and the DAPI reference spectrum recorded in vitro. Figure $3 \mathrm{f}$ shows the time trend of the PCC values retrieved from the WF depicted in Figure 3e. The evolution of the PCC over time reflects the dynamic nature of the system. In order to assess whether the observed PCCs were significantly higher than those yielded by random correlations, we calculated, in the same way, the PCC values for an endoscopy data set collected in the nuclei of unstained cells (negative control). The sample spectra extracted from the endoscopy control data set are provided in Figure S10 (details in the Supporting Informa- tion). The fingerprints show a remarkable spectral variability, originating from the complex intracellular environment, the presence of DNA proteins and enzymes, and the DNA polymeric nature, ${ }^{38}$ which render detailed peak assignment of native intracellular molecules risky and non-trivial. One way to overcome such uncertainty is to collect in vitro reference spectra of the molecules under study and perform correlation analysis, as undertaken in our work. The distribution of the $r$ values, calculated between the in vitro DAPI reference and the endoscopy negative control, exhibits a 95th percentile of 0.19 (Figure S11). Based on this outcome, only $r$ values higher than 0.19 were considered statistically significant and representative of the presence of DAPI in the system under study. The highest PCC value obtained for DAPI-stained cells in the WF shown in Figure $3 \mathrm{f}$ is 0.75 , which is generally accepted as satisfactory for the identification of molecules in complex systems, such as living cells. ${ }^{36,37}$ The spectrum linked to this value was compared with the in vitro SERS spectrum of DAPI (Figure $3 \mathrm{~g}$ ) to analyses and compare their respective spectral features, whereas more endoscopy fingerprints extracted around this $r$ value can be found in Figure S12. The fingerprint obtained from endoscopy measurements clearly displayed the main peaks of DAPI (details in the Supporting Information) and was consistent with the reference spectrum, as expected from the PCC calculations. Yet, a more in-depth assessment of the two spectra reveals intriguing details. Keeping in mind that DAPI binds to the DNA via the indole- $\mathrm{NH}$ proton and the amidinium groups, ${ }^{33-35}$ evidence of the DAPI-DNA complex formation comes from the drastic intensity decrease of the indole- $\mathrm{NH}$ deformation band at $739 \mathrm{~cm}^{-1}$ (Figure $3 \mathrm{~g}$, yellow band).

On the other hand, the peak associated with the amidinium moieties, at $1615 \mathrm{~cm}^{-1}$ (Figure $3 \mathrm{~g}$, orange band), remained clearly observable in the spectrum taken via endoscopy, presumably due to the strong Raman cross section of this vibration. ${ }^{39}$ Although maintaining a high intensity, a slight shift of this peak toward lower wavenumbers was noticeable, again probably associated with the DAPI-DNA complex formation. Such minor, but evident, spectral differences also explain the medium-to-high correlations observed in this case study.

To support the hypothesis that the spectral variations between the DAPI spectrum obtained from the nucleus (endoscopy data) and the one collected in vitro (reference) are due to the formation of the DAPI-DNA complex in the nucleus, we collected in vitro SERS spectra of the DNA-DAPI complex on AgNWs@Au, previously spin-coated on a glass coverslip. As expected, the obtained fingerprint presented the same alterations found during the endoscopy measurements (Figure S13). DNA peaks are absent in both the endoscopy data set and the in vitro fingerprints of the DAPI-DNA complex, likely because DAPI binds externally to the DNA. $^{33-35}$

Note that, upon probe insertion, contamination from the cellular membrane and/or cytoplasmic items might happen. However, the statistical screening performed in this study (PCC correlation) enables to extract exclusively the fingerprint most similar to that of the analyte, hence discarding the SERS signal coming from potential contaminants. Such a filtering is otherwise extremely complicated if no reference spectra are available for comparison (as in the case of intracellular native molecules).

Membrane. Endoscopy investigations on the cellular membrane were performed in a similar fashion. The membrane 
a)



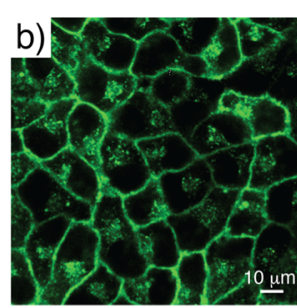
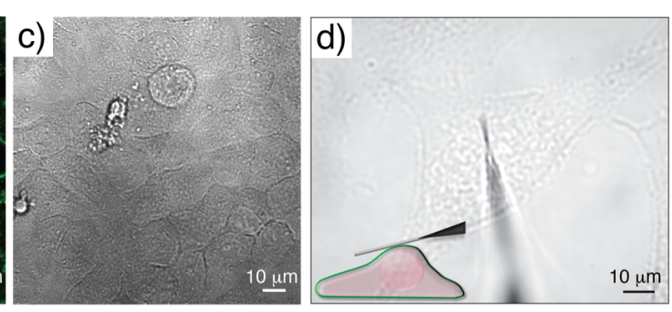
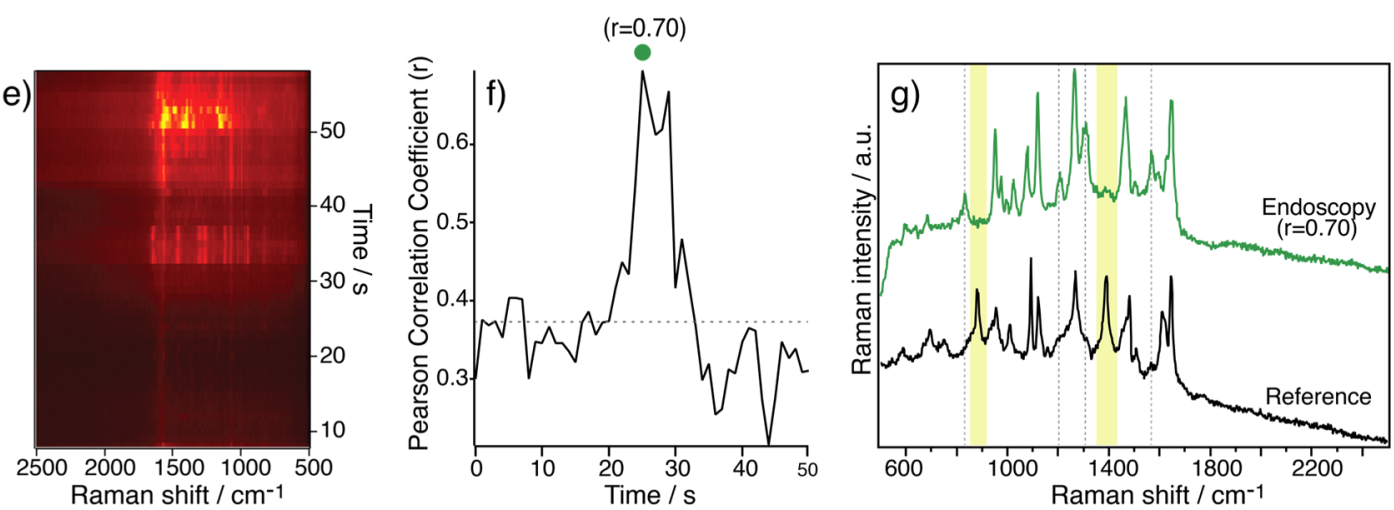

Figure 4. SERS endoscopy from the membrane of a DiO-stained HeLa cell. (a) Chemical structure of DiO and groups interacting with the phospholipid bilayer are highlighted in green. $(b, c)$ Confocal fluorescence (b) and transmission image (c) of HeLa cells stained with DiO. (d) Transmission image of the endoscopy probe placed onto the plasma membrane of a single HeLa cell. Inset: endoscopy experiment schematics. (e) Representative WF plot featuring the SERS spectra of DiO from the cell membrane over time. (f) Time trend of the PCCs calculated between each spectrum of the WF shown in (e) and the DiO SERS reference spectrum (in vitro). The horizontal line denotes the 95th percentile of the PCC distribution obtained for the control experiment $(r=0.37)$. Statistically significant $r$ values can be found above the percentile line. ( $g)$ SERS spectra of $\mathrm{DiO}$ from endoscopy measurements (top) and from in vitro measurements (reference) (bottom). Relevant Raman band changes are highlighted in yellow.

was stained with $\mathrm{DiO}$, a carbocyanine dye that incorporates into the phospholipid bilayer with its aliphatic chains (Figure $4 \mathrm{a}$, green background), while the chromophore moiety remains on the outer membrane surface. ${ }^{40,41}$ Figure $4 \mathrm{~b}, \mathrm{c}$ shows the fluorescence and transmission images of DiO-stained HeLa cells, respectively. The endoscopic probe was carefully placed on the membrane of a stained living HeLa cell (Figure 4d, Movie S2, and Figure S14).

SERS spectra were collected over time at $1 \mathrm{~s}$ integration from a point on the contact area between the AgNW@Au probe and the cellular membrane. A representative WF plot of the $\mathrm{DiO}$ signal over a time period of $50 \mathrm{~s}$ is reported in Figure 4e. As for DAPI, the spectra representative of the presence of $\mathrm{DiO}$ were selected through the estimation of PCC values. Here, the SERS spectrum of $\mathrm{DiO}$ obtained in vitro on AgNWs@Au was used as a reference. Figure 4f shows the time trend of the PCC values obtained for the WF depicted in Figure 4e. The PCC reached a maximum value of 0.70 at $25 \mathrm{~s}$, which falls in the high-medium correlation range.

In this case as well, in order to assess the statistical significance of the $r$ coefficients retrieved, we carried out endoscopy control measurements on the membrane of unstained cells (negative control) and we correlated them to the in vitro reference spectrum of DiO. Figure S15 reports a sample of SERS fingerprints extracted from the unstained membrane (details in the Supporting Information). Also, in this case, a remarkable spectral fluctuation is clearly observed and stems from the cellular rich environment previously addressed when discussing the nuclear negative control. The obtained distribution of $r$ values exhibits a 95th percentile of 0.37 (Figure S16). This higher value, compared with what was found for DAPI, stems from the larger amount of Raman peaks appearing both in the $\mathrm{DiO}$ and the unstained membrane fingerprints, which are more likely to overlap in a few regions.

Figure $4 \mathrm{~g}$ displays the spectrum associated with $r=0.70$ and the reference SERS spectrum of $\mathrm{DiO}$, whereas more fingerprints from the $\mathrm{DiO}$-stained membrane can be found in Figure S17. The spectrum obtained from the DiO-stained membrane displays the main vibrational modes of $\mathrm{DiO}$ (details in the Supporting Information) and comprehensively overlaps with the reference spectrum.

A more in-depth comparison between the $\mathrm{DiO}$ spectra collected from the membrane and the reference spectrum revealed the disappearance of the bands at 879 and $1386 \mathrm{~cm}^{-1}$. These bands are associated with the $-\mathrm{CH}_{2}$ and $-\mathrm{CH}_{3}$ deformation modes of the two long alkyl chains $\left(-\mathrm{C}_{18} \mathrm{H}_{37}\right)$ of $\mathrm{DiO}$ (Figure $4 \mathrm{~g}$, yellow bands). Considering that the hydrocarbon chains of $\mathrm{DiO}$ are spontaneously embedded in the phospholipid bilayer, ${ }^{40,41}$ the strong decrease of their Raman peaks intensity likely indicates the incorporation of the dye in the lipid bilayer. Another evidence of the $\mathrm{DiO}$ interaction with the lipid bilayer is given by the appearance of peaks at $830,1206,1304$, and $1567 \mathrm{~cm}^{-1}$, associated with the lipid head groups and proteins present on the outer membrane surface. ${ }^{42,43}$ In this case, the presence of membrane items in the SERS fingerprint arises from the fact that the $\mathrm{DiO}$ chromophore moiety is located in the outer membrane surface. These spectral differences explain the medium-to-high correlations obtained from the PCC calculations. Most importantly, they confirm the sensitivity of AgNW@Au probes toward chemical interactions occurring in the cells, between target molecules, such as DAPI or $\mathrm{DiO}$, and cellular organelles, such as cell nucleus or membrane. 


\section{CONCLUSIONS}

In summary, our work enlightens upon a simple and effective strategy, based on gold-etched AgNW endoscopy probes, for investigating the location and chemical interactions of exogenous molecules down to the single-cell level. We drastically improved the SERS performances of the AgNWbased endoscopic probes originally proposed by our group, rendering this strategy widely accessible, easily reproducible, and highly sensitive. This enhancement was performed by simply introducing gold nanostructures on the AgNWs via a GRR. Ultimately, we demonstrated the site specificity and applicability of these improved SERS probes for real-world applications by staining different cellular compartments and recording highly resolved fingerprints of their respective labels. The remarkable spectral sensitivity achieved additionally enabled the extraction of essential structural information of the target compounds, indicating that such an approach can be applied for unraveling molecular interactions between exogenous molecules (e.g., therapeutic drugs) and the cellular machinery. The platform developed herein clearly represents an innovative solution for uncovering a broad spectrum of drug interactions with biomolecular items, which might pave the way for crucial advances not only in fields such as drug development and disease treatment but also in analytical chemistry, forensics, and environmental sciences.

\section{ASSOCIATED CONTENT}

\section{s) Supporting Information}

The Supporting Information is available free of charge at https://pubs.acs.org/doi/10.1021/acs.analchem.0c04120.

Further experimental information regarding the AgNW synthesis, GRR, endoscopy probe SERS characterization, in vitro SERS references, PCC analysis and intracellular endoscopy measurements, and SERS peak assignment (PDF)

Endoscopy experiments in cell nucleus (AVI)

Endoscopy experiments on plasma membrane (AVI)

\section{AUTHOR INFORMATION}

\section{Corresponding Authors}

Beatrice Fortuni - Department of Chemistry, KU Leuven, 3001 Heverlee, Belgium; Email: beatrice.fortuni@ kuleuven.be

Hiroshi Uji-i - Department of Chemistry, KU Leuven, 3001 Heverlee, Belgium; Research Institute for Electronic Science, Hokkaido University, Sapporo 001-0020, Japan; ○ orcid.org/0000-0002-0463-9659; Email: hiroshi.ujii@ kuleuven.be

\section{Authors}

Monica Ricci - Department of Chemistry, KU Leuven, 3001 Heverlee, Belgium

Raffaele Vitale - Department of Chemistry, KU Leuven, 3001 Heverlee, Belgium; Laboratoire de Spectrochimie Infrarouge et Raman, Université de Lille, 59655 Lille, France; (i) orcid.org/0000-0002-7497-1673

Qiang Zhang - Research Institute for Electronic Science, Hokkaido University, Sapporo 001-0020, Japan

Yasuhiko Fujita - Department of Chemistry, KU Leuven, 3001 Heverlee, Belgium; (1) orcid.org/0000-0003-13021436
Shuichi Toyouchi - Department of Chemistry, KU Leuven, 3001 Heverlee, Belgium; (1) orcid.org/0000-0002-47718015

Gang Lu - Department of Chemistry, KU Leuven, 3001 Heverlee, Belgium; @ orcid.org/0000-0003-1722-0176

Susana Rocha - Department of Chemistry, KU Leuven, 3001 Heverlee, Belgium; 이이.org/0000-0003-1258-9396

Tomoko Inose - Research Institute for Electronic Science, Hokkaido University, Sapporo 001-0020, Japan

Complete contact information is available at:

https://pubs.acs.org/10.1021/acs.analchem.0c04120

\section{Author Contributions}

M.R. performed conceptualization, endoscopy measurements, and data analysis. R.V. performed the PCC analysis and critically revised the manuscript. Q.Z. and T.I. performed the SERS characterization; T.I. synthesized the AgNWs and carried out the TEM measurements. S.T. assisted the technical and optical aspects of the SERS measurements. S.R. contributed to the experiment design. Y.F. and G.L. crucially contributed to the development of the technique. B.F. and H.U.-i. supervised the experiments and led the project conceptualization. The manuscript was written through contributions of all authors. All authors have given approval to the final version of the manuscript.

\section{Funding}

National Natural Science Foundation of China (11974180), Key University Science Research Project of Jiangsu Province (17KJA150005), and Six Talent Peaks Project in Jiangsu Province (XCL-038).

\section{Notes}

The authors declare no competing financial interest.

\section{ACKNOWLEDGMENTS}

This work was funded by the Research Foundation-Flanders (grant nos G081916N, G0D4519N, G0A817N, and $1529418 \mathrm{~N})$, the KU Leuven Research Fund (C14/19/079, C14/15/053, and C14/16/053), and JSPS Kakenhi (JP17H03003 and JP20K21165 to H.U. JP19KK0136 to T.I.). The FWO scholarships to M.R. and B.F. (1S33117N and $12 \mathrm{X} 1419 \mathrm{~N}$, respectively) are greatly acknowledged. Q.Z. acknowledges the China Scholarship Council (CSC) for the doctoral scholarship. T.I. acknowledges Iketani Science and Technology Foundation, Nakatani Foundation, and the Research Program for CORE lab of "Five-star Alliance" in "NJRC Mater. \& Dev.". This collaborative work was supported by the JSPS Core-to-Core Program, A. Advanced Research Networks and the Photo-excitonix Project in Hokkaido University.

\section{REFERENCES}

(1) Kranz, C.; Eaton, D. C.; Mizaikoff, B. Anal. Bioanal. Chem. 2011, 399, 2309-2311.

(2) Chow, E. K. H.; Ho, D. Sci. Transl. Med. 2013, 5, 216 rv4.

(3) Thompson, M. Analyst 2004, 129, 671.

(4) Caruso, F.; Hyeon, T.; Rotello, V. M. Chem. Soc. Rev. 2012, 41, 2537-2538.

(5) Betty, Y. S.; Kim, M. D.; James, T.; Rutka, M. D.; Chan, W. C. W. N. Engl. J. Med. 2010, 17, 121-130.

(6) Souid, A.-K.; Tacka, K. A.; Galvan, K. A.; Penefsky, H. S. Biochem. Pharmacol. 2003, 66, 977-987.

(7) Octavia, Y.; Tocchetti, C. G.; Gabrielson, K. L.; Janssens, S.; Crijns, H. J.; Moens, A. L. J. Mol. Cell. Cardiol. 2012, 52, 1213-1225. 
(8) Gołuński, G.; Borowik, A.; Derewońko, N.; Kawiak, A.; Rychłowski, M.; Woziwodzka, A.; Piosik, J. Biochimie 2016, 123, 95-102.

(9) Larsen, A. K.; Escargueil, A. E.; Skladanowski, A. Pharmacol. Ther. 2000, 85, 217-229.

(10) Changenet-Barret, P.; Gustavsson, T.; Markovitsi, D.; Manet, I.; Monti, S. Phys. Chem. Chem. Phys. 2013, 15, 2937-2944.

(11) Lussier, F.; Missirlis, D.; Spatz, J. P.; Masson, J.-F. ACS Nano 2019, 13, 1403-1411.

(12) Wu, D.; Chen, Y.; Hou, S.; Fang, W.; Duan, H. ChemBioChem 2019, 20, 2432-2441.

(13) Niu, J. J.; Schrlau, M. G.; Friedman, G.; Gogotsi, Y. Small 2011, $7,540-545$.

(14) Vitol, E. A.; Orynbayeva, Z.; Bouchard, M. J.; AzizkhanClifford, J.; Friedman, G.; Gogotsi, Y. ACS Nano 2009, 3, 3529-3536.

(15) Geßner, R.; Rösch, P.; Petry, R.; Schmitt, M.; Strehle, M. A.; Kiefer, W.; Popp, J. Analyst 2004, 129, 1193-1199.

(16) Polwart, E.; Keir, R. L.; Davidson, C. M.; Smith, W. E.; Sadler, D. A. Appl. Spectrosc. 2000, 54, 522-527.

(17) Mullen, K. I.; Carron, K. T. Anal. Chem. 1991, 63, 2196-2199.

(18) Brasiliense, V.; Park, J. E.; Chen, Z.; Van Duyne, R. P.; Schatz,

G. C. J. Raman Spectrosc. 2020, 1-9.

(19) Vitol, E. A.; Orynbayeva, Z.; Friedman, G.; Gogotsi, Y. J. Raman Spectrosc. 2012, 43, 817-827.

(20) Singhal, R.; Orynbayeva, Z.; Sundaram, R. V. K.; Niu, J. J.; Bhattacharyya, S.; Vitol, E. A.; Schrlau, M. G.; Papazoglou, E. S.; Friedman, G.; Gogotsi, Y. Nat. Nanotechnol. 2011, 6, 57-64.

(21) Lu, G.; De Keersmaecker, H.; Su, L.; Kenens, B.; Rocha, S.; Fron, E.; Chen, C.; Van Dorpe, P.; Mizuno, H.; Hofkens, J.; Hutchison, J. A.; Uji-I, H. Adv. Mater. 2014, 26, 5124-5128.

(22) Netzer, N. L.; Qiu, C.; Zhang, Y.; Lin, C.; Zhang, L.; Fong, H.; Jiang, C. Chem. Commun. 2011, 47, 9606-9608.

(23) Sun, Y.; Xia, Y. J. Am. Chem. Soc. 2004, 126, 3892-3901.

(24) Lin, H.; Ohta, T.; Paul, A.; Hutchison, J. A.; Demid, K.; Lebedev, O.; Van Tendeloo, G.; Hofkens, J.; Uji-I, H. J. Photochem. Photobiol., A 2011, 221, 220-223.

(25) Moran, C. H.; Rycenga, M.; Zhang, Q.; Xia, Y. J. Phys. Chem. C 2011, 115, 21852-21857.

(26) Eilers, P. H. C. Anal. Chem. 2003, 75, 3631-3636.

(27) Orendorff, C. J.; Gole, A.; Sau, T. K.; Murphy, C. J. Anal. Chem. 2005, 77, 3261-3266.

(28) Tiwari, R.; Jain, P.; Asati, S.; Haider, T.; Soni, V.; Pandey, V. J. Drug Delivery Sci. Technol. 2018, 48, 383-392.

(29) Hurley, L. H. Nat. Rev. Cancer 2002, 2, 188-200.

(30) Alves, A. C.; Ribeiro, D.; Nunes, C.; Reis, S. Biochim. Biophys. Acta, Biomembr. 2016, 1858, 2231-2244.

(31) Bernardes, N.; Fialho, A. M. Int. J. Mol. Sci. 2018, 19, 3871.

(32) Tan, L. T.-H.; Chan, K.-G.; Pusparajah, P.; Lee, W.-L.; Chuah,

L.-H.; Khan, T.-M.; Lee, L.-H.; Goh, B.-H. Front. Pharmacol. 2017, 8, 12.

(33) Špačková, N.; Cheatham, T. E.; Ryjáček, F.; Lankaš, F.; Van Meervelt, L.; Hobza, P.; Šponer, J. J. Am. Chem. Soc. 2003, 125, 1759-1769.

(34) Mildner, B.; Metz, A.; Chandra, P. Cancer Lett. 1978, 4, 89-98.

(35) Granzhan, A.; Kotera, N.; Teulade-Fichou, M.-P. Chem. Soc. Rev. 2014, 43, 3630-3665.

(36) Biscaglia, F.; Rajendran, S.; Conflitti, P.; Benna, C.; Sommaggio, R.; Litti, L.; Mocellin, S.; Bocchinfuso, G.; Rosato, A.; Palleschi, A.; Nitti, D.; Gobbo, M.; Meneghetti, M. Adv. Healthcare Mater. 2017, 6, 1700596.

(37) Biscaglia, F.; Quarta, S.; Villano, G.; Turato, C.; Biasiolo, A.; Litti, L.; Ruzzene, M.; Meneghetti, M.; Pontisso, P.; Gobbo, M. Mater. Sci. Eng. C 2019, 103, 109762.

(38) Barhoumi, A.; Zhang, D.; Tam, F.; Halas, N. J. J. Am. Chem. Soc. 2008, 130, 5523-5529.

(39) Prochazka, M. Surface-Enhanced Raman Spectroscopy; Biological and Medical Physics, Biomedical Engineering; Springer International Publishing: Cham, 2016.

(40) Axelrod, D. Biophys. J. 1979, 26, 557-573.
(41) Honig, M. G.; Hume, R. I. Trends Neurosci. 1989, 12, 333-341. (42) Fogarty, S. W.; Patel, I. I.; Martin, F. L.; Fullwood, N. J. PLoS One 2014, 9, No. e106283.

(43) Richter, M.; Hedegaard, M.; Deckert-Gaudig, T.; Lampen, P.; Deckert, V. Small 2011, 7, 209-214. 